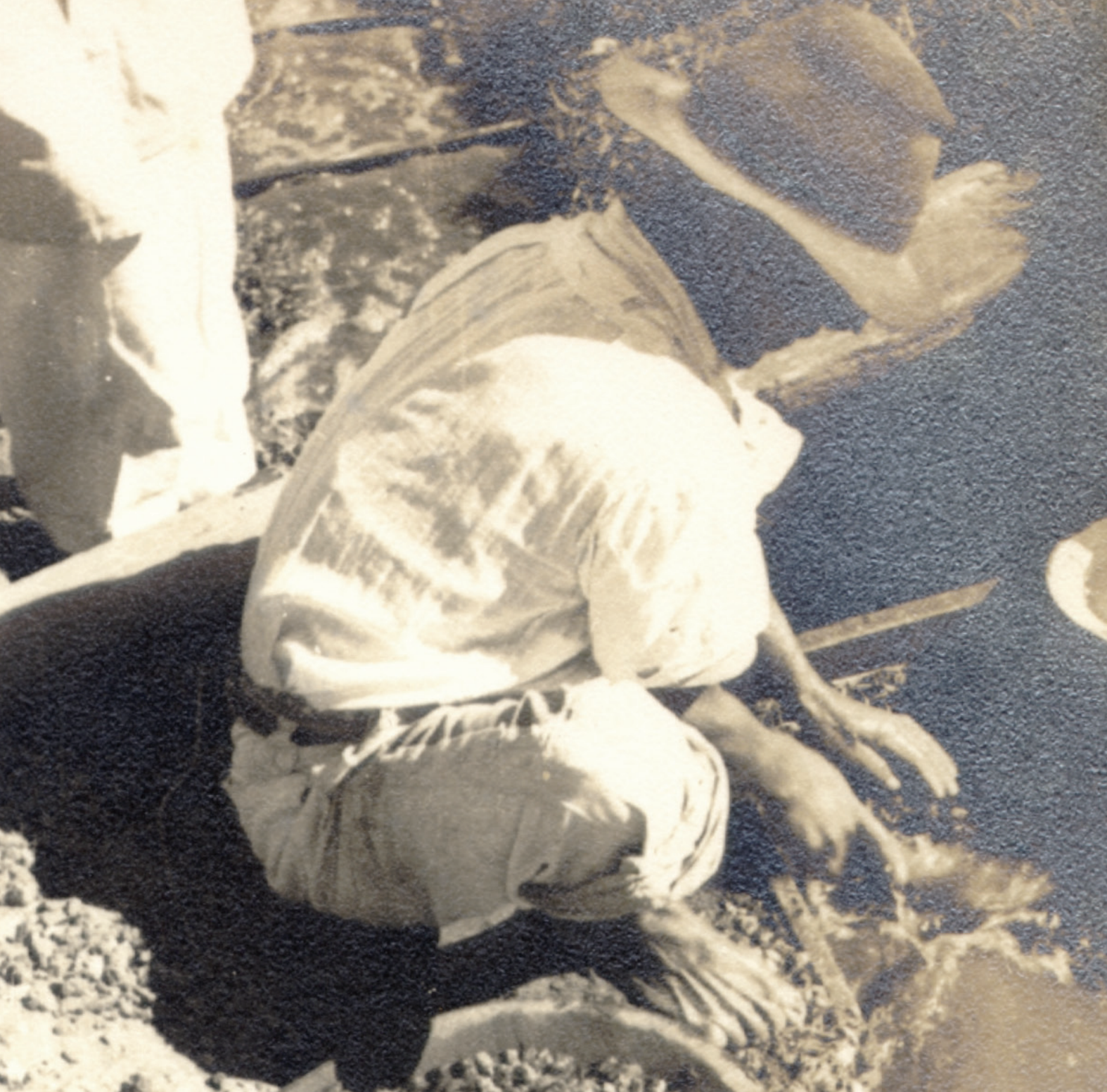

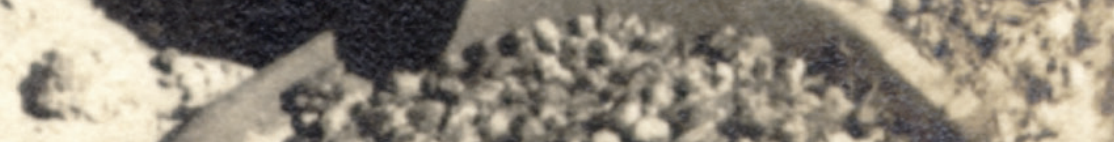

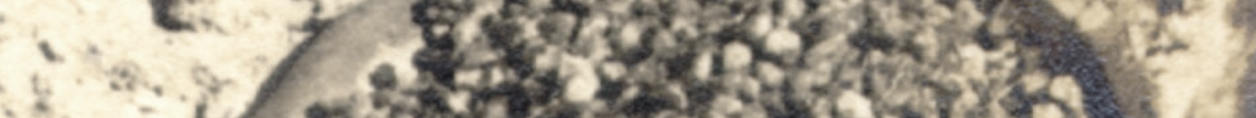

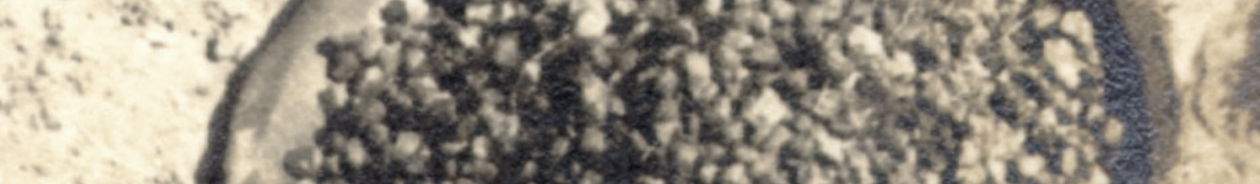

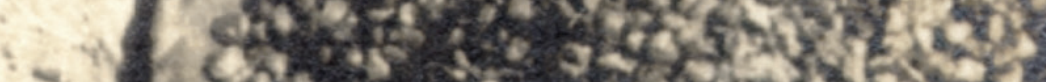

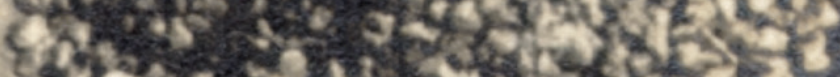

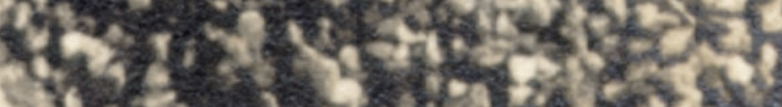

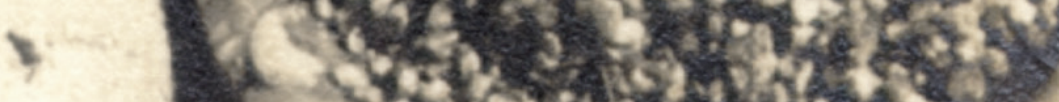

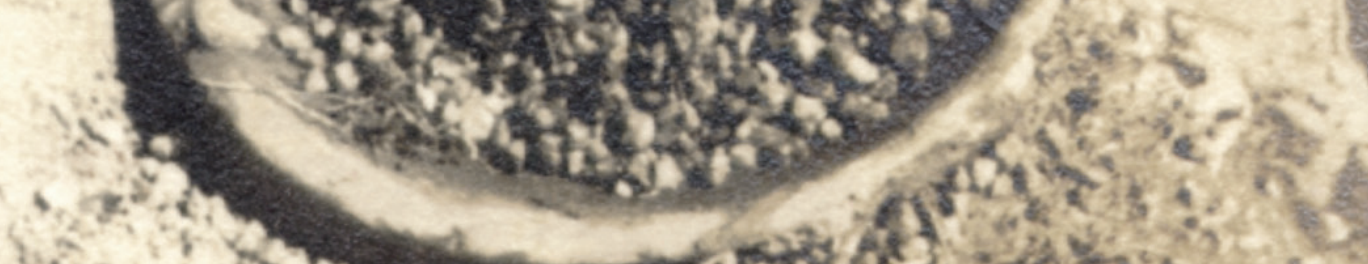

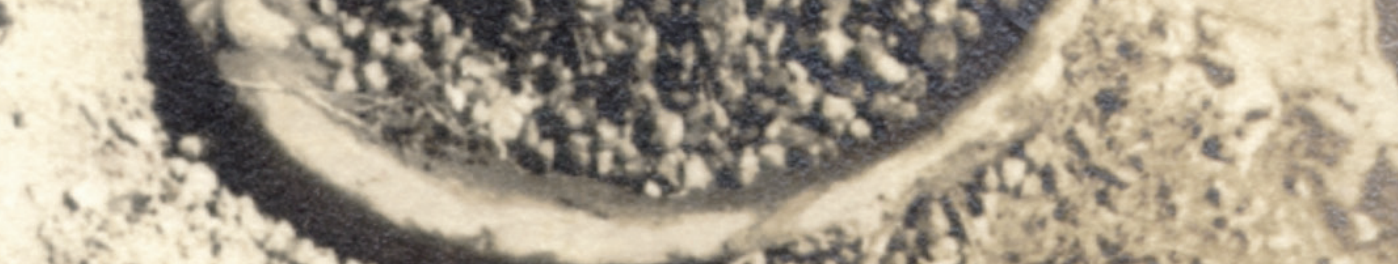

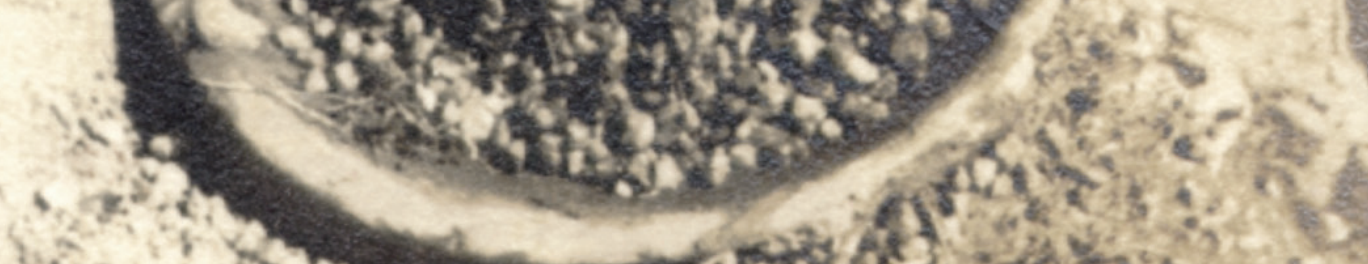


TSP

Universidade de São Paulo

Prof. Dr. Marco Antonio Zago Reitor

Prof. Dr. Vahan Agopyan Vice-reitor

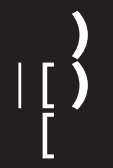

Instituto DE Estudos Brasileiros

Prof. ${ }^{a}$ Dr. ${ }^{a}$ Maria Angela Faggin Pereira Leite Diretora

Prof. ${ }^{\mathrm{a}}$ Dr. ${ }^{\mathrm{a}}$ Marina de Mello e Souza Vice-diretora 


\section{Revista do Instituto de Estudos Brasileiros}

ISSN 0020-3874

número 58, 2014 jan./jun.

Comissão Editorial

Denilson Lopes Silva (UFRJ) Rio de Janeiro, BR

Gustavo Alejandro Sorá (UNC) Córdoba, AR

Jaime Tadeu Oliva (IEB-USP) São Paulo, BR

Paulo Teixeira Iumatti (IEB-USP) São Paulo, BR

Pedro Meira Monteiro (Princeton U.) Princeton, EUA

Randal Johnson (UCLA) Los Angeles, EUA

Walter Garcia (IEB-USP) São Paulo, BR

Editores Responsáveis

Jaime Tadeu Oliva (IEB-USP)

Walter Garcia (IEB-USP)

\section{Editor Adjunto}

Paulo Teixeira Iumatti (IEB-USP)

Dossiê O Brasil no período Lula - organização Jaime Tadeu Oliva (IEB-USP)

\section{Colaboração}

Alexandre de Freitas Barbosa (IEB-USP)

Produção

Aline Khoury

Assistentes editoriais

Fernanda Rodrigues Rossi

José Hermes Martins Pereira

Lia Marques (estagiária)

\section{Equipe de apoio}

Regina Mayumi Aga

Editoração eletrônica

Iris Fabrin Sototuka (estagiária)

\section{Colaboraram neste número}

Ana Paula Cavalcanti Simioni (IEB-USP)

Flávia Camargo Toni (IEB-USP)

Ana Maria Naito Horiuchi (preparação de texto)

Fernando Toledo (trad. língua alemã)

Luíz H. Mello (preparação em língua inglesa)

Luciana Araújo (preparação de texto)

Bruno José Pereira, Juliana Dourado, Lydia M.

Cintra, Suellen Freitas, Vinicius S. Almeida, Wipsley

M. dos Santos (revisão)

\section{Projeto gráfico}

Homem de Melo \& Troia Design

Impressão:

\section{Distribuição:}

Editora 34

R. Hungria, 592 | 01455-000 São Paulo SP

(11) 38166777 | vendas@editoraz4.com.br
Conselho Consultivo

Adrián Gorelik

Universidade Nacional de Quilmes, Bernal, AR

Barbara Weinstein

Universidade de Nova lorque, Nova lorque, EUA

Carlos Augusto Calil

Universidade de São Paulo, São Paulo, BR

Carlos Sandroni

Universidade Federal de Pernambuco, Recife, BR

Ettore Finazzi-Agrò

Universidade de Roma La Sapienza, Roma, IT

Fernanda Arêas Peixoto

Universidade de São Paulo, São Paulo, BR

Heloisa Maria Murgel Starling

Universidade Federal de Minas Gerais, Belo Horizonte, BR

João Cezar de Castro Rocha

Universidade Estadual do Rio de Janeiro, Rio de Janeiro, BR

Jorge Coli

Universidade Estadual de Campinas, Campinas, BR

Luiz Felipe de Alencastro

Universidade de Paris-Sorbonne, Paris, FR

Manuel Villaverde Cabral

Universidade de Lisboa, Lisboa, PT

Maria Cecilia França Lourenço

Universidade de São Paulo, São Paulo, BR

Maria Ligia Coelho Prado

Universidade de São Paulo, São Paulo, BR

Maria Lucia Bastos Kern

Pontificia Universidade Católica do Rio Grande do Sul, Porto Alegre, BR

Peter Burke

Emmanuel College Cambridge, Cambridge, RU

Regina Zilberman

Universidade Federal do Rio Grande do Sul, Porto Alegre, BR

Ricardo Augusto Benzaquen de Araújo

Pontificia Universidade Católica do Rio/ Instituto Universitáriode Pesquisas do Rio de Janeiro, Rio de Janeiro, BR

Rodolfo Nogueira Coelho de Souza

Universidade de São Paulo, São Paulo, BR

Sergio Miceli

Universidade de São Paulo, São Paulo, BR

Walnice Nogueira Galvão

Universidade de São Paulo, São Paulo, BR

Instituto de Estudos Brasileiros

Edifício Brasiliana, Praça do Relógio Solar, 342

Cidade Universitária

05508-050, São Paulo - SP, Brasil

(11) 30911149

www.ieb.usp.br 


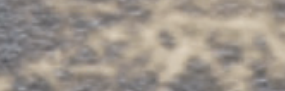

$\frac{2 x}{2 x^{2}}$

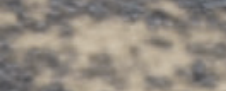

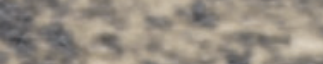

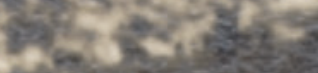

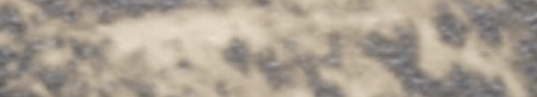

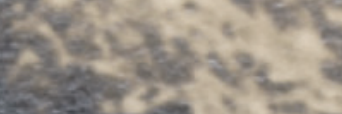
Q

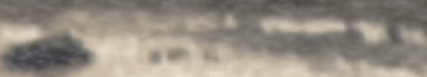

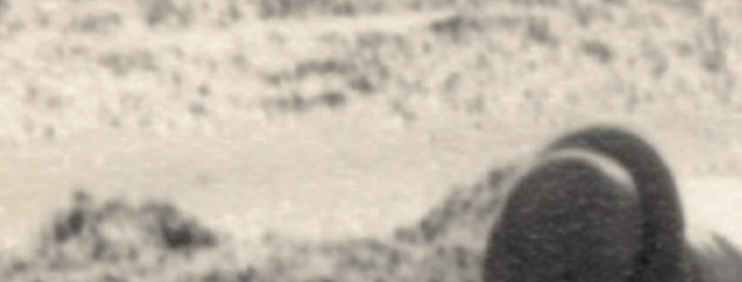

48

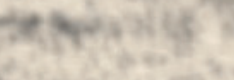

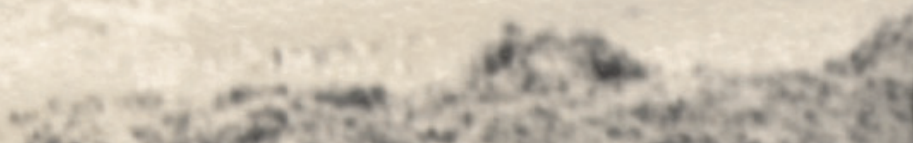

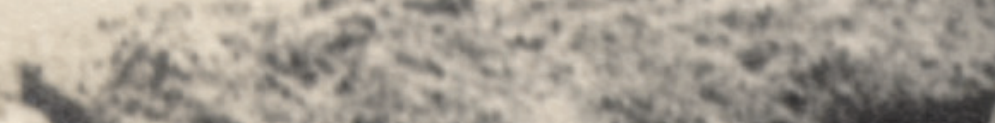

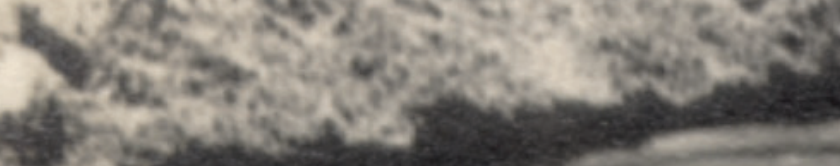
axistis

\section{t.}
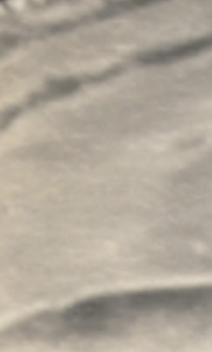

\section{$2 .+\operatorname{lin}^{2} x^{2}$

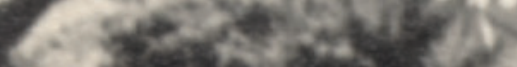

$\therefore \lim ^{2}$

$-8$

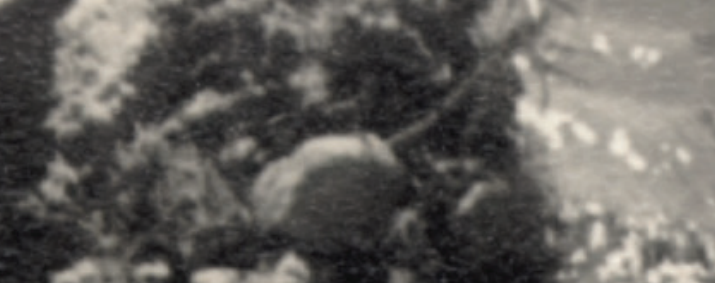

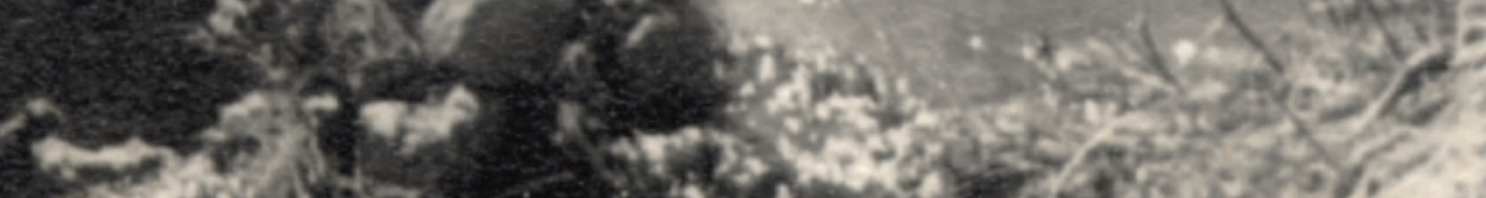




\section{SUMÁRIO}

II Editorial

Artigos

I7 A periferia como obra: modernidades excêntricas a re-arranjos Luso-tropicalistas

Roвerto VEcCHI

35 Esquerdas, política e cultura no Brasil (1950-1970):

um balanço historiográfico

Marcos Napolitano

SI Curral de reses, curral de almas: introdução à urbanização

dos "Certoens" das capitanias do Norte (séculos XVII-XIX)

EsDras Arraes

79 "Chicago" no Brasil: a importância da redescoberta da cidade e da "raça"

FranK ECKARDT

105 Da relevância pública dos espaços livres: um estudo sobre metrópoles e capitais brasileiras

Eugenio Fernandes Queiroga

Dossiê O Brasil No PERíodo LULA

135 "Era Lula", "desenvolvimentismo" e as desigualdades estruturais

Alexandre de Freitas Barbosa

I37 Política Cultural em tempos de democracia: a Era Lula Lia CALABRE

I57 A Era Lula/Tamborzão: política e sonoridade Guillermo Caceres, Lucas Ferrari e Carlos Palombini

209 Política para a educação superior no governo Lula: expansão e financiamento Cristina Helena Almeida de Carvalho

245 Cidades na era Lula: o lento abandono dos processos participativos

Fernando Luiz Lara 
263 A Era Lula e sua questão econômica principal: crescimento, mercado interno e distribuição de renda ANDRÉ M. BIANCARELLI

RESENHAS

29I Pemedebismo renitente

Roberto Pereira Silva

299 Borracha, gás e tiro: impressões sobre Cidades rebeldes Christian GiLioti

DocumentaÇÃo

313 David Vygódski: modernismo e política no Brasil e na União Soviética

Bruno Barretto Gomide

323 Vanguardas documentadas: o concretismo de Theon Spanudis

Bárbara Sesso Carneiro

NotícIAs

$33 I$ IEB produz série "Galáxias", sobre como pensar o Brasil hoje, em parceria com o SESC TV

333 Notícias do Serviço Educativo do IEB-USP

Errata

$343 \quad$ Errata - Número 57 


\section{TABLE OF CONTENTS}

II Editorial

Articles

I7 Periphery as a Work: Eccentric Modernities and Lusophone-Tropical Rearrangements

Roberto Vecchi

35 Politics and Culture in the Brasilian Left:

a Historiographical Balance

Marcos Napolitano

SI Cattle's Corral, Soul's Corral:

Introduction to Urbanization of the "Certoens" of the North Captaincies (17th - 19th centuries)

Esdras Arraes

79 "Chicago" in Brazil: The Rediscovered Importance of the City and "Race"

Frank ECKardt

Io5 About Public Importance of the Open Spaces:

the Case of Brazilian Metropolises and Brazilian Capitals Eugenio Fernandes Queiroga

Dossier Brazil in Lula's age

135 "Lula's age", "developmentalism" and the structural inequalities

Alexandre de Freitas Barbosa

137 Cultural Policy in Democratic Times: The Lula Age

Lia Calabre

I57 The Age of Lula/Tamborzão: Politics and Sonority

Guillermo Caceres, Lucas Ferrari e Carlos Palombini

209 Higher Education Policy in the Lula Government:

Expansion and Funding

Cristina Helena Almeida de Carvalho

245 Cities in Lula's Era: The Slow Death of Participatory Processes

Fernando Luiz Lara 
263 Lula's Era and its Main Economic Question:

Growth, Domestic Market and Income Distribution

ANDRÉ M. BiANCARELLI

BOOK REVIEWS

29 The Resistent Pemedebismo

Roberto Pereira Silva

299 Baton, Burners and Shots: Impressions of Cidades Rebeldes'

Christian GiLioti

Documents

313 David Vygódski: modernism and politics in Brazil and in the Soviet Union

Bruno Barretto Gomide

323 Documented Vanguards: Concretism of Theon Spanudis Bárbara Sesso Carneiro

News

$33 I$ IEB produces audiovisual series 'Galaxia' to discuss comtemporary Brazil, in partnership with SESC TV

333 News from Serviço Educativo do IEB-USP

Erratum

343 Erratum - Number 57 


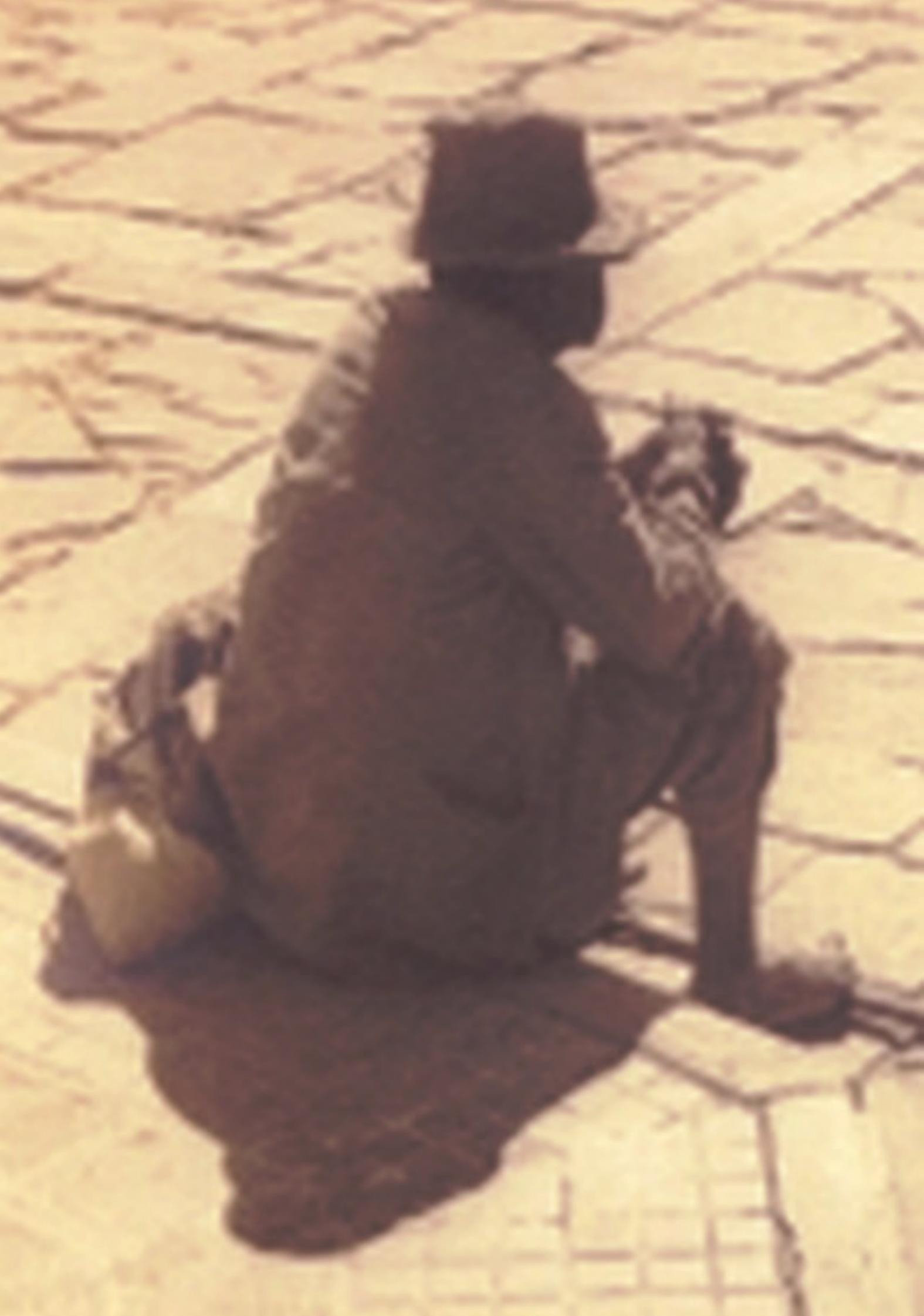




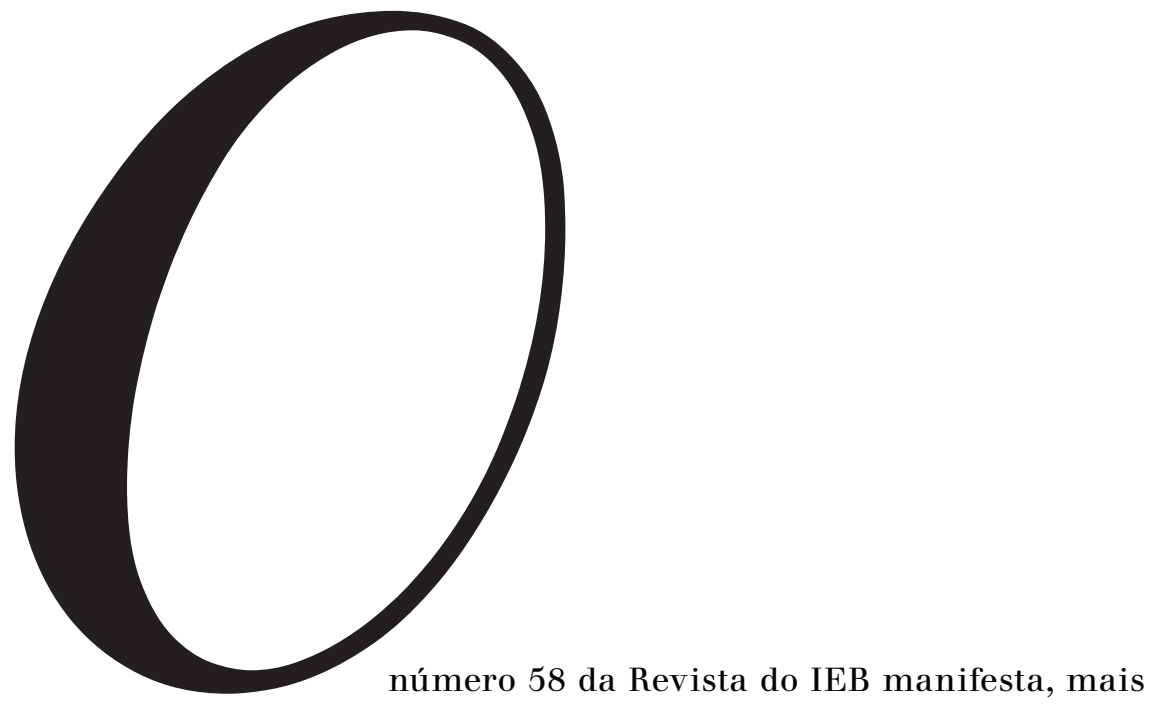
uma vez, a proposta multidisciplinar dessa publicação, cuja linha editorial projeta a própria vocação do instituto, que organiza nessa chave as suas atividades acadêmicas - curadoria de acervos, pesquisas e cursos de graduação e pós-graduação; o Brasil enquanto tema polariza essas atividades, assim como o teor geral dos diversos números da RIEB ao longo de sua história.

Certamente os artigos da Revista podem ser lidos numa chave disciplinar, mas a trama interdisciplinar que eles suscitam resulta numa dimensão da leitura que nos parece bastante produtiva. Nesse número, particularmente, essa trama resultou de uma estratégia editorial que procurou destacar uma reflexão diversificada sobre o Brasil contemporâneo, daí o convite para que o volume seja apreciado no seu conjunto. Não é sem propósito o apelo, pois nos parece hora de avaliarmos o provável prejuízo que há na crescente fragmentação e descaracterização das revistas acadêmicas, algo facilitado pelos novos suportes de publicação e pelos consequentes mecanismos de busca.

Como resultado dos novos parâmetros implementados na Revista do IEB, esse número traz um conjunto mais amplo de artigos. São dez artigos somados a duas resenhas, duas publicações de documentos e algumas notícias do IEB. Nesses termos, esse volume é bem substancioso.

Podemos dividir os artigos em dois conjuntos. O primeiro traz uma seleção de cinco textos cuja origem é bastante sui generis, reunindo colaboradores de diversas formações e pertencentes a várias instituições nacionais e internacionais.

A despeito da ausência, nessa primeira parte, de uma orientação temática, o conjunto conta com três artigos que discutem a questão urbana no Brasil. O artigo de Esdras Arraes (USP) investe na história de 
uma situação regional do processo de urbanização brasileiro no período entre os séculos XVII ao XIX, especificamente nas zonas interiorizadas que compõe o atual nordeste brasileiro. O Brasil urbano contemporâneo inspira os outros dois artigos. Eugenio Fernandes Queiroga (USP) nos traz uma contribuição para debatermos parâmetros que são fundamentais para vida, mas que são subestimados: a importância dos espaços livres no interior das metrópoles brasileiras para a constituição dos espaços públicos, e por consequência de uma vida pública mais relevante. Por sua vez, o sociólogo urbano Frank Eckardt (Universidade Bauhaus, Weimar-Alemanha) nos surpreende com uma enfática defesa do legado da chamada Escola de Chicago de Robert E. Park nos estudos urbanos brasileiros. Essa produção destacava o racismo como um ingrediente importante na constituição da desigualdade brasileira e, embora atacada na época de sua publicação, ainda seria estimulante para os estudos das cidades brasileiras contemporâneas.

As outras duas contribuições a esse conjunto correspondem a textos de reflexão no âmbito da crítica cultural. O artigo de Roberto Vecchi (Universidade de Bolonha, Itália) discute as recentes elaborações a respeito do conceito de periferia, diante da persistência da ideologia do luso-tropicalismo em suas formulações. Para ele, é necessário manter a crítica alerta contra esses dispositivos ideológicos ocultos na teoria póscolonial. Já o historiador Marcos Napolitano (USP) pretende um balanço crítico da trajetória da arte engajada de esquerda no Brasil, entre 1950 e 1970. Seu objetivo central é analisar as lacunas e contradições da análise historiográfica provocadas pela excessiva dependência desta em relação à memória produzida pelos sujeitos históricos em disputa nesse campo.

O segundo conjunto de artigos compõe um dossiê orientado pelos editores, interessados, nesse caso, em expor nas páginas da revista as tensões sociais (e suas possíveis interpretações) do Brasil contemporâneo. O dossiê se intitula “O Brasil no período Lula". A abordagem adotada foi a da verticalização temática trabalhada por especialistas das áreas da cultura, da música, da economia, da educação e da questão urbana. Evidentemente que esse estilo de abordagem não é o único possível e tem várias limitações. No entanto o tema do dossiê é preferencialmente tratado junto à opinião pública, e mesmo no mundo acadêmico, como certa totalidade. E nesse caso (nessa escala de abordagem), nem sempre as visões sobre ruptura ou continuidade em relação ao cenário social anterior são claras. Interessante notar, nesse aspecto, o contraste com as interpretações temáticas, que com mais desembaraço notam as permanências e as mudanças de rumo. 
Os artigos do dossiê “O Brasil no período Lula” respondem diferentemente sobre a existência ou não de mudanças estruturais no país. Contudo, respondem com segurança. O texto de Lia Calabre (FCRB) faz um balanço das políticas culturais durante os dois mandatos do Presidente Lula (2003-2010) e enfatiza a implementação de parâmetros de política cultural mais democráticos e participativos que alcançaram o patamar mais elevado que o país conheceu nessa área. Portanto, ela responde positivamente à tese de mudanças estruturais nesse período. Por outro lado, Carlos Palombini (UFMG), Guillermo Caceres (UFC) e Lucas Ferrari (UFMG), ao associarem o desenvolvimento e a morfologia de três bases rítmicas características de três décadas sucessivas do funk carioca a eventos políticos, percebem no comprometimento do primeiro presidente petista com a militarização da segurança pública um dado de regressão, ou de persistência, de posturas repressivas em relação às classes populares e à sua cultura. Com a mesma frustração crítica, Fernando Lara (Universidade do Texas, EUA), já ressoando em seu texto as surpreendentes manifestações populares de junho de 2013, afirma que as cidades brasileiras não estão melhores que há dez anos, apesar das ressalvas sobre outras áreas de atuação do governo. Principalmente, o artigo lamenta certa regressão no impulso democrático para a vida das cidades, bem recepcionada pelo estado anteriormente e desestimulada no período tratado, marcado pela maior participação dos cidadãos nas decisões.

O artigo de Cristina Helena Almeida de Carvalho (UnB) trata de maneira favorável uma das dimensões da política educacional, a saber: a expansão e o financiamento da educação superior com o presidente Lula à frente do executivo. Para ela, a política educacional consubstanciou-se na combinação de crescimento intensivo e extensivo acompanhado do acréscimo de recursos ao segmento federal. E houve nesse sentido uma ruptura (no mínimo uma aceleração) em relação a políticas anteriores. Finalmente, o artigo de André Martins Biancarelli (Unicamp) procura contrapor-se à crítica dominante sobre o agravamento nas condições econômicas no Brasil nos anos de 2012 e 2013, que ele reputa em parte como alarmismo injustificável, sem deixar de notar o contraste com o otimismo reinante até o final do governo Lula. Para ele, os pilares principais da questão econômica sob esse governo que são o crescimento, o desenvolvimento do mercado interno e a justiça social são parâmetros que ainda devem balizar as discussões nessa área, pois já desempenharam um papel relevante na história recente do país.

Está aí a controvérsia entre os especialistas das diversas áreas e entre essa visão temática e verticalizada e o que costuma predominar 
nas interpretações de caráter mais abrangente. Está aí desenhado, nesse dossiê, um ângulo interessante da complexidade do Brasil contemporâneo que merece mais do que ser exposto, já ser objeto de uma discussão. É o que faz, abrindo a seção, Alexandre Barbosa (IEB), no provocativo texto "Era Lula", "Desenvolvimentismo" e Desigualdades Estruturais.

Na seção Resenhas, duas obras que direta ou indiretamente tratam das manifestações populares de junho de 2013 são objeto de análise. Na resenha de Roberto Pereira Silva (Unifal), o trabalho de Marcos Nobre Imobilismo em movimento é tratado como repertório para o entendimento de certa renitência na vida política brasileira, que nos leva a uma tão falada crise de representatividade. E vinculado mais estreitamente às manifestações populares de 2013, o livro coletivo Cidades Rebeldes é avaliado segundo esse foco, por Christian Gilioti (USP).

Em Documentação, Bruno Gomide (USP) revela uma inesperada aproximação entre um escritor russo, David Vygodski, e os modernistas brasileiros, a partir de uma crítica sobre Literatura Brasileira descoberta em seus arquivos pessoais. Igualmente com base em arquivos pessoais, Bárbara Carneiro (USP) traz um levantamento do Fundo Theon Spanudis no Arquivo do IEB, revelando os desafios de se lidar com uma documentação sobre múltiplos assuntos - da psicanálise à arte contemporânea - e escrita em diversas línguas.

Reiterando o convite para um proveito maior da trama interdisciplinar que esse número proporciona, esperamos que ela suscite ao leitor uma boa leitura.

Jaime Oliva, Paulo Iumatti e Walter Garcia Editores 